\title{
Circuit
}

Musiques contemporaines

\section{Maryvonne Kendergi (1915-2011) : hommage et mémoire}

\section{Louise Bail}

Volume 22, numéro 3, 2012

Viva la musica ! Ana Sokolović

URI : https://id.erudit.org/iderudit/1014230ar

DOI : https://doi.org/10.7202/1014230ar

Aller au sommaire du numéro

Éditeur(s)

Les Presses de l’Université de Montréal

ISSN

1183-1693 (imprimé)

1488-9692 (numérique)

Découvrir la revue

Citer ce document

Bail, L. (2012). Maryvonne Kendergi (1915-2011) : hommage et mémoire.

Circuit, 22(3), 67-72. https://doi.org/10.7202/1014230ar d'utilisation que vous pouvez consulter en ligne.

https://apropos.erudit.org/fr/usagers/politique-dutilisation/ 


\section{ACTUALITÉS}

\section{Maryvonne Kendergi (1915-2011): hommage et mémoire}

Louise Bail

Maryvonne Kendergi fut une figure marquante de l'évolution de la culture musicale vivante au Québec. Par sa présence aux micros de Radio-Canada, elle a su initier le public à la musique contemporaine et établir un réseau très riche d'échanges entre les compositeurs québécois et les plus illustres penseurs du $\mathrm{Xx}^{\mathrm{e}}$ siècle. Dans la foulée de ses cours de musique canadienne à l'Université de Montréal, elle a également su mettre à la disposition de ses étudiants les œuvres significatives du répertoire contemporain qui émergeaient de l'actualité musicale.

Les grandes institutions gouvernementales, universitaires et culturelles de Montréal, du Québec, du Canada et de la France lui ont décerné les plus hautes distinctions, rendant ainsi hommage à ses aptitudes, à sa passion et à sa détermination ${ }^{1}$.

Aux harmoniques multiples que déclenche ce mot de «présences» vient s'ajouter la dimension irremplaçable des voix et des visages de nos invités: créateurs et interprètes, artistes et exégètes. Ils sont eux-mêmes générateurs d'autres présences que font surgir leurs propos.

- Maryvonne Kendergi, La Semaine à Radio-Canada, 1961.

Des trois termes qui qualifient son ancrage au Québec - nom, voix, présence - , la présence est celui dont nous nous souviendrons le plus. La veille de son départ définitif, le 26 septembre 2011, alors que je m'arrêtais aux soins palliatifs du Centre hospitalier de St. Mary, lors de ma promenade quotidienne dans le quartier qui est devenu le mien et qu'elle a tellement aimé habiter, j'ai eu l'impression étrange de recueillir son dernier et long regard. Impression, bien sûr, puisque ses nièces, Annette et Denise Kouri, ont

\footnotetext{
1. Parmi la pléthore de prix et d'honneurs qu'elle a récoltés, mentionnons: Officier de l'Ordre du Canada (1980), récipiendaire de la Médaille du Conseil canadien de la musique (1981), récipiendaire du Prix Lynch-Staunton du Conseil des Arts du Canada (1982), Chevalier de l'Ordre national du Québec (1985), Officier de l'Ordre des Arts et des Lettres de la République française (1985), récipiendaire du prix de musique Calixa-Lavallée de la Société Saint-JeanBaptiste de Montréal (1985), membre de l'Académie des Grands Montréalais (1988), membre de la Société royale du Canada (1990), récipiendaire du prix hommage du Conseil québécois de la musique pour l'ensemble de sa carrière (2000).
} 


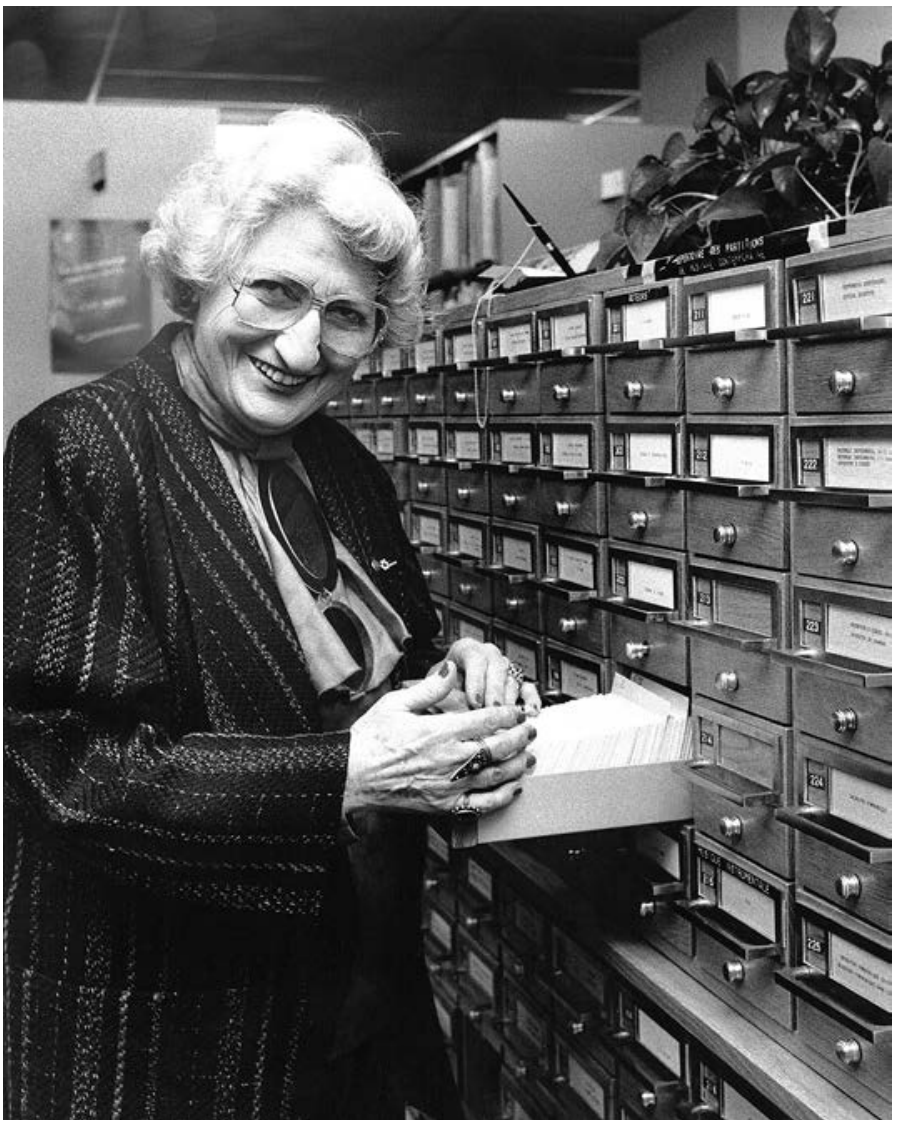

2. Deux ans et demi avant son décès, Marius Constant m'avait reçue chez lui, à Paris, afin que je puisse lui transmettre les bons vœux de son amie probablement eu cette ultime chance de lui dire adieu. Ce regard que j'ai recueilli - comme c'était étrange! - ne m'était pas adressé. J'avais l'impression qu'il ne voyait plus personne ici-bas. Cette femme qui conjuguait au présent le passé et l'avenir dans un seul geste, celle qui ne vivait ni d'un passé qui n'est plus ni d'un avenir qui n'est pas, n'était déjà plus là. Dorénavant elle ne subsisterait que dans nos mémoires.

Je me suis souvent interrogée sur la signification de ces deux lignes que Marius Constant avait envoyées à l'éditeur pour paraître en exergue de ses mémoires: «Soleil : quand tu parais. Nuit: quand tu disparais. » Je me disais que Marius Constant était trop malade pour en écrire davantage et que cette épigraphe elliptique était peut-être même le fait d'un cerveau qui déclinait². 
Comme maintenant ces deux lignes m’apparaissent éclairantes! Elles disent tout Maryvonne Kendergi : soleil de la présence que fut sa vie et nuit de la mort qui vide notre espace d'une porte-parole talentueuse, sensible, convaincue et toujours ouverte aux voies de l'avenir.

Avant tout présente aux autres, son existence est ainsi résumée dans le titre de ses mémoires, La Musique en partage, emprunté à une série d'entretiens radiophoniques de cinq heures qu'elle avait accordés à Françoise Davoine (2001) et qu'elle tenait à utiliser pour leur publication.

Être présent aux autres dans le partage, cela aura été de parler des autres et de se mettre à leur service toute sa vie. Elle l'a fait en mettant en relation les uns avec les autres, toujours très à l'aise dans le rôle d'«intermédiaire» dont elle fit très vite glisser le sens vers celui d'«entremetteuse », terme qu'elle employa un nombre incalculable de fois avec fierté, toujours en l'assortissant d'un brin d'humour impertinent. Cela aura été de saisir toutes les occasions qui s'offraient à elle pour travailler au développement du milieu musical (par le micro, l'enseignement universitaire, la défense de la musique vivante, la constitution de fonds pour la recherche en musique du Québec, etc.). Cela aura été de s'engager envers les milieux culturels et communautaires chrétiens qu'elle fréquenta à Paris, à Gravelbourg et dans son quartier de Côte-des-Neiges. Vers la fin de sa vie, alors que retraitée bénéficiant de plus de loisirs, elle se dévouera aux plus faibles et aux assistés sociaux (les Petits Frères des Pauvres), aux prisonniers (réminiscence sans doute du temps de la guerre)... Le mouvement de lutte contre la grande pauvreté et l'exclusion sociale ATD Quart-Monde, qu'elle intégra à cette époque en parfaite affinité avec son action militante et discrète dans le mouvement missionnaire laïque Ad Lucem3, la ramènera à ses objectifs d'origine, aux valeurs et aux moteurs qui donnèrent un sens à sa vie: servir sa foi chrétienne et la culture française par le moyen le plus pacifique qui soit, par la musique.

Dire qu'elle fut une présence, c'est aussi se référer aux titres des multiples émissions qu'elle signa et qu'elle anima ou auxquelles elle prêta son concours à la Société Radio-Canada - Présence de l'autre, Présences polonaises, Présence de l'art —, sans compter ces autres titres qui suggéraient des rencontres sur le ton parfois d'une complicité toute maternelle (Tante Marie des tout-petits, station $\mathrm{CFRG}^{4}, 1953$ ), qui proposaient des entretiens qu'elle désirait amicaux (Les Jeudis de Maryvonne), qui relataient des découvertes (Le Billet de Maryvonne), des interviews chaleureuses de personnalités rencontrées au hasard de reportages qui démontraient un humanisme formé aux plus hauts standards culturels (Propos, Carrefour, Musiciens d'aujourd'hui, Parallèlement...).
3. Maryvonne Kendergi devint membre d'Ad Lucem le 8 décembre 1940, le jour de l'Immaculée Conception, l'une de ses grandes dévotions mariales. À l'époque, la mission du mouvement international et interracial Ad Lucem, créé à Lille en 1932 par le docteur Louis Aujoulat, était un groupe d'avant-garde et utopique - parce qu'il désirait transcender toutes les religions - dont la mission était de porter le message du Christ en observant la spiritualité de Charles de Foucault, une spiritualité qui s'exerçait dans le silence et qui se vivait parmi les "infidèles", sans forcer leur conversion au christianisme.

4. "Crois, Façonne, Rayonne, Garde". CFRG est une station appartenant à l'Association communautaire fransaskoise de Gravelbourg (ACFG). 
5. Ses collaborations à Radio-Canada s'échelonneront bien au-delà de sa prise de retraite de l'université en 1981. Elle couvrira de façon régulière les activités de la scène culturelle montréalaise et les productions du Festival international du film sur l'art (FIFA), notamment à l'émission CBF-Bonjour...
Un nom se forge dans l'agir. Et son nom fut très vite associé à sa voix. C'est d'ailleurs de cette voix dont se souviennent encore les auditeurs de Radio-Canada. De la radio, elle en avait fait avant d'arriver à Montréal, alors qu'elle faisait connaître régulièrement les résidents de la Cité universitaire de Paris aux échanges internationaux de la Radiodiffusion française dans une série d'émissions Vos étudiants chez nous (rediffusion par CKAC-Montréal, entre 1948 et 1952). Ses mémoires racontent son passage de quatre années à Gravelbourg où elle eût son premier vrai baptême des ondes radiophoniques. À Montréal (automne 1956), où elle s'arrêta pour remplir une promesse, elle fut retenue par Marc Thibault, alors directeur du réseau français radiophonique de Radio-Canada, à qui, raconte-t-elle, elle devra l'orientation des deux derniers tiers de sa vie. Il lui obtint l'émission hebdomadaire Festival du dimanche qui devint, après deux ans, Festivals européens (1958-1963). Puis ce fut une floraison de participations et de collaborations à la radio et à la télévision où elle essayait de dire la musique, qu'elle situait dans un contexte culturel large et très riche, à un auditoire de plus en plus fasciné par ces nouvelles musiques et par les anciennes aussi, échos lointains des festivals extraordinaires d'où elle rapportait les commentaires et confidences de personnages non moins captivants 5 .

Son enseignement à la Faculté de musique de l'Université de Montréal (1967-1981) sera en quelque sorte le prolongement de ses activités de documentaliste-commentatrice-conceptrice à la radio. La matière était la même. Les étudiants réentendaient dans leurs cours d'histoire et de musique vivante les voix de leur époque: celles des Xenakis, Stockhausen, Messiaen, Varèse, Poulenc... Toutes ces voix, ils les associaient à la sienne, en interaction avec d'autres dont la présence animait ses cours de musique canadienne: les Papineau-Couture, Pépin, Morel, Tremblay, Garant... Ces compositeurs et interprètes deviendront les personnages des Musialogues (1970-1983) qui firent de la faculté un essaim bourdonnant d'abeilles industrieuses, un atelier où chaque étudiant avait sa place dans la grande chaîne des tâches qu'elle distribuait, où chacun travaillait avec fierté à la connaissance et à la promotion de la musique du $\mathrm{Xx}^{\mathrm{e}}$ siècle.

"J'ai apporté au Canada sincèrement et honnêtement, dira-t-elle un jour, tout ce que j'avais de connaissances et d'expériences» (La Presse, 1975). Je crois que ces propos dans leur simplicité résument bien ce que fut cette femme de grande stature qui donna plus qu'elle ne reçut. Des études restent certainement à faire sur sa traversée des esthétiques du $\mathrm{XX}^{\mathrm{e}}$ siècle et sur l'impact qu'a eu son travail sur la modernité musicale au Québec. Il serait également opportun de s'interroger sur la pérennité d'une ouvre conservée 
sur support d'enregistrement sonore. Grâce à la vigilance de la professeure Marie-Thérèse Lefebvre et du travail d'archivage du compositeur Mario Gauthier, les étudiants en musicologie, récipiendaires des bourses du Fonds Maryvonne Kendergi, pourront à leur aise effectuer des recherches sur le "phénomène Kendergi», ses archives sonores et l'ensemble de ses documents ayant été versés aux Archives de l'Université de Montréal.

\section{RÉFÉRENCES SUPPLÉMENTAIRES}

BaIL, Louise (2002), Maryvonne Kendergi: la musique en partage. Montréal, HMH Hurtubise ${ }^{6}$. Bail Milot, Louise (dir.) (1985), «Musialogue: Maryvonne Kendergi », Les Cahiers de $l^{\prime} A R M u Q, n^{\circ} 5$ (mai), p. 1-887.

\section{REPÈRES CHRONOLOGIQUES - MARYVONNE KENDERGI}

1915,15 août

1918

1928

1929-1933

1933, juin

1937

1938, juin

1940, juin

1942, juin

1945, 31 octobre

1947, 30 juin

1948, hiver

1949, janvier

1952, 3 novembre

$195^{2-1956}$
Maryvonne Kendergi (Kendirjian) naît durant le génocide arménien à Aïntab (en Cilicie), aujourd'hui Gaziantep (Turquie), de parents arméniens.

La famille quitte pour Alep.

Maryvonne obtient son brevet d'études primaires.

Étudie le piano et les matières musicales académiques à l'École normale de musique de Paris, sous la férule de Nadia Boulanger.

Obtient son diplôme d'enseignement, section piano, de l'École normale de musique; commence une carrière de concertiste au Moyen-Orient (en Syrie et au Liban).

Retourne à Paris, loge au Foyer international des étudiantes et reprend des études à l'École normale de musique.

Réussit l'épreuve de piano de la licence d'enseignement et entreprend la licence de concert en piano avec Alfred Cortot.

Obtient la licence complète d'enseignement.

Obtient la licence libre ès lettres constituée de quatre certificats: juin 1939, certificats d'esthétique et d'histoire de la musique; septembre 1940, certificat de littérature; juin 1942, certificat d'histoire de l'art.

Quitte le Foyer international pour s'établir au Collège franco-britannique de la Cité universitaire de Paris; y organise des activités culturelles.

Se fiance à Chartres avec Pierre Laforte; fiançailles qui seront rompues à l'automne.

Déménage du Collège franco-britannique à la Fondation des États-Unis où on la nomme «Head of Cultural Section »; en cette qualité devient déléguée au Centre culturel international de la Cité universitaire.

Contribue à mettre sur pied, avec des résidents de la Fondation des ÉtatsUnis, le Club musical, qui deviendra la section musicale du Centre culturel international.

Quitte la France pour rejoindre ses parents chez sa sœur Aimée à Gravelbourg, en Saskatchewan.

Travaille à Gravelbourg; à CFRG (Gravelbourg), puis au collège de Gravelbourg comme enseignante d'initiation à la musique.
6. Cet ouvrage a été publié dans la collection Musique, dirigée par Lyse Richer, des "Cahiers du Québec".

7. Ce dossier, présenté sous forme des Musialogues, a été constitué dans le cadre des manifestations de la Journée internationale de la musique de 1981. Il fut commandé par la Société de musique contemporaine du Québec et réalisé par Louise Bail avec l'aide de Christiane Plamondon et de Jeannine Barriault. Le document a été produit avec l'assistance de l'Association pour l'avancement de la recherche en musique du Québec (ARMUQ, aujourd'hui Société québécoise de recherche en musique, SQRM) et l'appui financier du Conseil des arts du Canada. Mario Gauthier possède un exemplaire annoté d'informations ultérieures à 1985 par Maryvonne Kendergi.

8. Ces repères chronologiques sont une version revue et corrigée à partir de celle publiée dans le livre Maryvonne Kendergi: la musique en partage (2002), p. 23-24. 
1955, été

1956, 8 septembre

$195^{6-1962}$

1961, été

1962, printemps

$1965-1966$

$1966-1981$

1970

1981

1981-2008
Passe l'été à Paris avant de revenir pour une dernière année à Gravelbourg.

Quittant Gravelbourg pour retourner à Paris, elle s'arrête à Montréal; à Radio-Canada, est retenue par Marc Thibault, le directeur du réseau français de Radio-Canada, qui la convainc de rester en lui offrant une série d'émissions qui débuteront le 30 septembre sous le titre de Festival du dimanche.

Après deux ans, Festival du dimanche devient Festivals européens; c'est le début d'une longue carrière à la radio qui perdurera plusieurs années après sa prise de retraite.

Dans le cadre du Festival de Montréal, participe à l'organisation de la Semaine internationale de musique actuelle aux côtés de Pierre Mercure et de Serge Garant.

Avec Pierre Mercure, se rend à New York pour interroger Edgard Varèse sur Debussy en vue d'une première apparition à la télévision, à l'émission L'Heure du concert.

Participe à la fondation de la Société de musique contemporaine du Québec (SMCQ), dont le premier concert aura lieu le 15 décembre 1966.

À la demande de Jean Papineau-Couture, intègre le corps professoral de la Faculté de musique de l'Université de Montréal en même temps que Serge Garant; y enseigne la musicographie préparant aux carrières musicales parauniversitaires et inaugure les premiers cours de musique canadienne dans une université canadienne.

Instaure les Musialogues (rencontres musicales) qui animent le milieu universitaire jusqu'en 1983 et, au-delà de cette date, la scène montréalaise lors d'événements musicaux, notamment en s'entretenant avec des invités de l'Orchestre symphonique de Montréal.

Prend sa retraite de la Faculté de musique; y reçoit le titre de professeure émérite, entre autres pour son apport au rayonnement de l'Université de Montréal.

Poursuit ses contributions philanthropiques et caritatives dans différents secteurs de la vie sociale; siège à des conseils d'administration d'organismes culturels (dont le Conseil des arts de la Communauté urbaine de Montréal [1985-1991]); couvre pour la radio des événements de la scène culturelle (entre autres, le Festival international du film sur l'art [FIFA]); à la demande de Radio-Canada, soumet trois séries de treize entretiens marquants avec des compositeurs et des interprètes d'ici et d'ailleurs (Trésors d'archives, émissions réalisées par Jean Boivin [1986, 1988 et 1992]); en 2008, quitte son appartement du Rockhill à Côte-des-Neiges en confiant à Mario Gauthier le classement et le dépôt de ses archives à l'Université de Montréal.

2011, 27 septembre Décède au Centre hospitalier de St. Mary. 